\title{
Quantification of patellar tendon shortening in a patient with cerebral palsy
}

\author{
Manuela Galli ${ }^{1}{ }^{2}$, Veronica Cimolin ${ }^{1}$, Sara Vimercati ${ }^{1}$, Giorgio Albertini' ${ }^{2}$, Reinald Brunner ${ }^{3}$ \\ 'Department of Electronics, Information and Bioengineering, Politecnico di Milano, Milano - Italy \\ 2IRCCS "San Raffaele Pisana" -Tosinvest Sanità SpA, Roma - Italy \\ ${ }^{3}$ Children's University Hospital Basel (UKBB), Basel - Switzerland
}

\begin{abstract}
Purpose: In the last few years the use of patellar tendon shortening procedure appears to give good results for the treatment of crouch gait in Cerebral Palsy (CP) patients. It offers a secondary approach in cases where conventional hamstrings lengthening failed. The evidence for the effectiveness of knee extensor shortening is poor and only one study quantitatively assessed the effects of this technique performed in addition to other procedures in CP patients. In this case study we used Gait Analysis (GA) to quantify the effects of patellar tendon shortening for the management of crouch gait persisting after hamstring lengthening.

Methods: Crouch persisted seven years after a first multilevel procedure including hamstring lengthening in a 15-year old male. Gait was assessed over time (before and from six to 18 months after knee extensor shortening) with Gait Analysis (GA). Results: Before treatment the patient walked slowly and with a high duration stance phase; sagittal kinematics revealed excessive hip and knee flexion and ankle dorsiflexion during the whole gait cycle. After surgery, data showed progressive significant improvements at all lower limb levels during the follow-ups considered; gait became more physiologic and lower limb joint kinematics improved overall.

Conclusions: The results demonstrated that addressing the other side and shortening the antagonists may be a valuable option to treat crouch gait in patients with CP especially if this persists after hamstring and knee extension surgery.
\end{abstract}

Key words: Cerebral palsy, Crouch, Gait analysis, Patellar tendon shortening

Accepted: December 19, 2011

\section{INTRODUCTION}

One of the most common and resistant conditions to treat in patients with spastic diplegia secondary to Cerebral Palsy (CP) is crouch gait, characterized mainly by increased knee flexion gait during the stance phase. Some factors can contribute to this pattern, including hamstring and/or psoas tightness because of spasticity or contracture, lever arm dysfunction, weakness, and impaired balance (1-3). Once crouching reaches a certain level of severity, knee flexion and associated symptoms may progress rapidly because of high stresses at the knee and failure of the knee extensor mechanism. Knee pain because of increased patellofemoral compressive forces frequently develop as well and may even result in a fracture of the patella. As the cause of crouch gait is multifactorial and difficult to characterize precisely, treatment is controversial.

Conservative treatments, such as stretching of spastic knee flexors, strengthening of knee and hip extensors, fitting of orthosis, fail to correct this problem in most cases, and the choice of orthopedic procedures is also difficult. Most traditional surgical procedures address muscle tightness and contracture but not muscle insufficiency. In addition, because most patients with persistent crouch gait have commonly had previous interventions, including muscle lengthening procedures, it is necessary to consider alternative methods of intervention, such as muscle shortening (3-5). Recently, Young et al (6) discussed sagittal gait patterns in children with spastic diplegia, focusing the attention on the knee, as well as the concept of the "dose" of surgery required to correct different gait pathologies. The concept of dose seems to be useful in the consideration of the management of knee dysfunction.

In the last few years the use of patellar tendon shortening procedure appears to give good results for the treatment of crouch gait. The evidence for the effectiveness of this procedure is poor; to the best of our knowledge only one study quantitatively assessed the effects of this technique performed in addition to other procedures in $\mathrm{CP}$ patients using 3D Gait Analysis (GA) (5). These authors transposed the tibial tubercule distally in order to retain 
the extensors. In this case study, in contrast, the patellar ligament was duplicated and thus shortened which is more convenient in the growing skeleton.

In order to improve the knowledge on the effects of this procedure, in this case study we used GA to quantify the effects of patellar tendon shortening for the management of crouch gait.

\section{MATERIALS AND METHODS}

\section{Patient presentation}

The patient was a 15-year-old adolescent (weight: $67 \mathrm{~kg}$, height: $167 \mathrm{~cm}$ ) affected by diplegic CP, with severe crouch gait. In 2000 he walked with flexed knees and abduction feet, and had initial contact with the toes. At this time a bilateral Grice arthrodesis was carried out in order to stabilize the feet and to improve triceps function. At the same time both Achilles' tendons were lengthened, and in order to improve knee extension, lengthening of the left hamstrings was added. However, this surgery did not resolve the problem. Seven years later, he presented with severe crouch which led to anterior knee pain and rapidly deteriorating gait and function. He had constant physiotherapy but refused to carry orthoses. The clinical data are presented in the results section.

\section{Treatment}

The aim of treatment was to gain passive and active full knee extension in order to enable full active knee control during gait. As a first step, only the semitendinosus tendons were released in order to improve knee extension. Intra-operatively the gain was only $10^{\circ}$, hence leaving another $15^{\circ}-20^{\circ}$. In order to avoid a possible knee hyperextension because of stretching out of the remaining hamstrings and posterior knee structures the planned supracondylar extension femoral osteotomy was postponed and a three month period of stretching followed this minor intervention. During this time passive knee extension recovered completely and the need for the supracondylar osteotomy was withdrawn. During the rehabilitation period, a muscle strength training programme improved general muscle strength as well as the strength of the plantar flexors to grade 3 and 4 respectively (Manual Muscle Strength Test). The active extension lag of the knee, however, remained and so did the crouch gait pattern. For this reason the knee extensors were shortened bilaterally in a second intervention. The principle of this procedure was to duplicate the patellar tendon in order to get the patella down and to increase knee extensor tension. The leg was draped allowing for free movement of the leg during surgery. The patellar tendon was exposed from origin to insertion through a longitudinal median incision. The knee was held in maximal flexion in order to obtain tension on the patellar ligament, and the ligament was divided in the frontal plane leaving $1 / 3$ to $1 / 2$ of the previous thickness. For this purpose a small scalpel (no 20 blade) with a long handle was pushed through the ligament followed by a clamp which then was moved up and down inside of the ligament. This allowed a fully blunt dissection leaving two bundles. The knee was extended and the anterior bundle divided horizontally through the middle. A first no. 6 Vicryl pierced the proximal third of the intact posterior bundle, several times up on the medial side to the insertion at the patella and down on the lateral side back to the same level as medial. In a similar way another no. 6 Vicryl was interwoven in each half of the dissected ventral bundle of the ligament, leaving two stumps fixed with sutures. The first suture (of the intact posterior bundle) was now completed pushing the strong needle of the no. 6 suture through the tibial tubercle. The posterior bundle was plicated by tightening this suture. The two stumps of the anterior bundle were reinserted by similar transosseous fixation at the equivalent bony ligament insertion at the tibial tubercule and the distal patellar pole respectively, which resulted in an overlay of the two stumps of the anterior (ventral) bundle. Therefore, the posterior part of the ligament remained intact in case of failure of the suture but the ventral part allowed sufficient shortening of up to $4 \mathrm{~cm}$. The wound was closed. The leg was then held in extension for six weeks allowing only $40^{\circ}$ of flexion during physiotherapy. Only after this time did intensive physiotherapy include mobilization exercise to regain mobility and active muscle strength training was performed.

\section{Assessment}

The patient was examined clinically and with instrumented 3D GA in a multidisciplinary team before (PRE session), six months (POST1 session), 12 months (POST2 session), and 18 months (POST3 session) after the operation.

The 3D GA was performed using a 12-camera optoelectronic system (ELITE2002, BTS S.p.A., Milan, Italy) with passive markers positioned according to Davis (7), for movement assessment kinematics, two force platforms (Kistler, $\mathrm{CH}$ ), for movement kinetic assessment, and a synchronic video system (BTS S.p.A., Milan, Italy). Anthropometric data were collected and passive markers were placed at anatomic landmarks. The patient walked barefoot at his self-selected and convenient speed along a $10 \mathrm{~m}$ walkway. At least sev- 


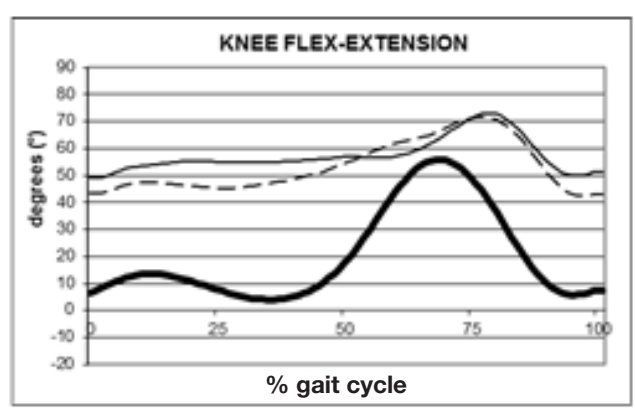

(a)

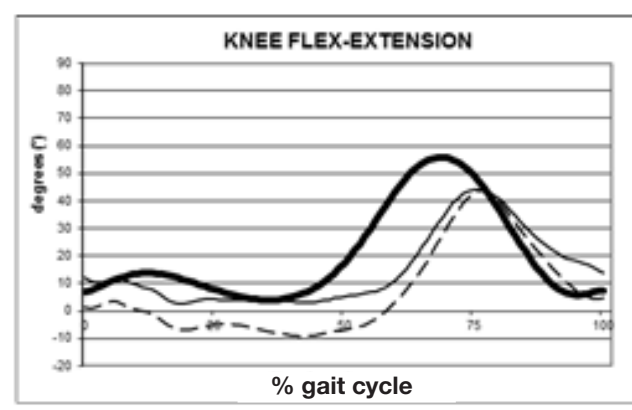

(c)

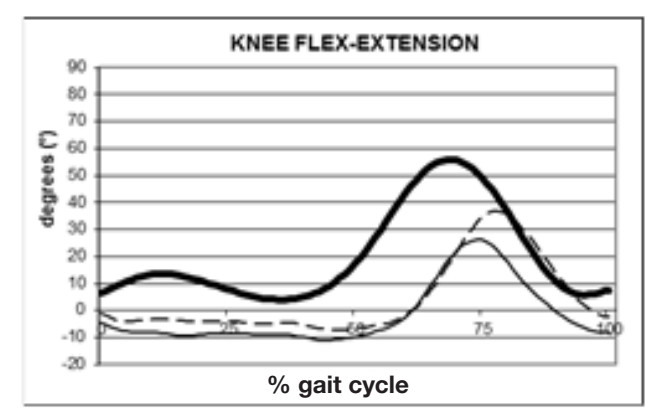

(b)

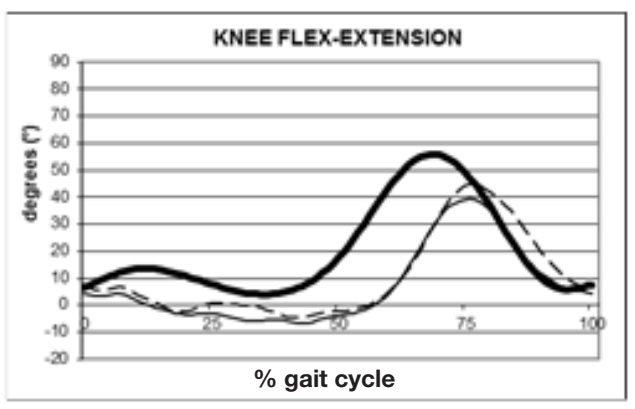

(d)
Fig. 1 - Knee flex-extension angle of a trial of the patient analyzed (solid line: right limb; dashed-line: left limb) in PRE (a), POST1 (b), POST2 (c), and POST3 (d) sessions and normative range of control group (thick lines) are reported. en trials were recorded during each session. The data were computed according to Davis using Euler angles (7).

Some parameters were identified and calculated from kinematic and kinetic data (ankle moment and power) on the sagittal plane: spatio-temporal parameters, joint-angles values at a specific instant of the gait cycle, and on the sagittal plane of movement and peaks in ankle moment and power graphs (Tab I).

The mean values (standard deviation) of kinematic and kinetic parameters are shown in detail in Table II, Table III, and Table IV, in PRE, POST sessions, and for aged matched normals (control group, CG). Figure 1 presents the knee flex-extension plot of all patient sessions of the patient.

The gait data of the patient was compared to CG.

\section{Statistics}

A one-way inter-group analysis of variance (ANOVA) was applied to compare PRE, POST1, and POST2 sessions; the assumptions of the ANOVA model were tested by assessing the fit of the observed data to normal distribution (Kolmogorov-Smirnov test) and homogeneity of variances (Levene's test). Specific effects were assessed by means of the post-hoc comparisons of means (Bonferroni test). Null hypotheses were rejected when probabilities were below 0.05.

\section{RESULTS}

PRE session

\section{Clinical assessment}

The patient walked in crouch. Clinical assessment revealed a knee flexion contracture of only $30^{\circ}$ right and $25^{\circ}$ left. Ankle dorsiflexion came up to $15^{\circ}$, and there were $10^{\circ}$ of hip flexion contracture which was regarded secondary to the knee. The patient showed very good motor control with mild spasticity only. On x-ray there was an extremely high riding patella bilaterally.

Gait analysis

The subject walked slowly, long stance phase and reduced step length compared to normal values.

The knee joint was characterized by an excessive flexion during stance (IC and min St indices) attaining maximally $45^{\circ}$ left and $50^{\circ}$ right at mid stance and to a lesser extent in swing (Max Sw index) phase, showing a limited range of motion bilaterally (Fig. 1 a).

The ankle joint was dorsiflexed during the whole gait cycle with physiologic joint excursion in stance phase.

The hip showed excessive flexion at initial contact (IC) and reduced extension in midstance; the range of motion, however, was close to normality, although the data were shifted towards flexion.

The mean pelvic tilt was close to normality but was characterized by a higher range of motion than CG. 


\begin{tabular}{ll}
\hline Gait Parameter & Description \\
\hline $\begin{array}{l}\text { Spatio-temporal parameters } \\
\text { Speed }(\mathrm{m} / \mathrm{s})\end{array}$ & $\begin{array}{l}\text { mean velocity of progression } \\
\text { \% stance }(\% \text { of gait cycle })\end{array}$ \\
$\begin{array}{l}\text { Step length }(\mathrm{cm}) \\
\text { Kinematics }\end{array}$ & $\begin{array}{l}\text { longitudinal distance from one foot strike to the next one } \\
\text { Pelvic Tilt }\end{array}$ \\
ROM & $\begin{array}{l}\text { the range of motion at pelvic joint on the sagittal plane during the gait cycle, calculated as the difference } \\
\text { mean value of motion at pelvic joint on the sagittal plane during the gait cycle }\end{array}$ \\
Mean value & $\begin{array}{l}\text { value of hip flexion on the sagittal plane at initial contact, representing the position of hip joint at the begin- } \\
\text { ning of gait cycle } \\
\text { IC Flex-Extension }\end{array}$ \\
minimum of hip flexion on sagittal plane in stance phase, representing the extension ability of hip during \\
this phase of gait cycle \\
the hip range of motion on the sagittal plane during the gait cycle, calculated as the difference between the \\
maximum and minimum values of the plot
\end{tabular}

Knee Flex-Extension

IC

Min in St

Max in Sw

ROM

Ankle Dorsi-Plantarflexion

IC

Max in St

Min in St

Max in Sw

$\mathrm{ROM}$ in St

$\underline{\text { Kinetics }}$

Ankle Dorsi-Plantarflexion Moment

Max in St

Ankle Power

Max in St

Kinetic data revealed a physiologic ankle plantarflexor moment but a low peak of ankle power generation at the push-off bilaterally at increased dorsiflexion indicating plantarflexor weakness. ning of gait cycle this phase of gait cycle this phase of gait cycle at the beginning of gait cycle ability of ankle joint at toe-off value of knee flexion on sagittal plane at initial contact, representing the position of knee joint at the begin-

minimum of knee flexion on sagittal plane in mid-stance, representing the extension ability of knee during

peak of knee flexion on sagittal plane in swing phase, representing the flexion ability of knee joint during

the knee range of motion on the sagittal plane during the gait cycle, calculated as the difference between the maximum and minimum values of the plot

value of the ankle joint angle on sagittal plane at the initial contact, representing the position of knee joint

peak of ankle dorsiflexion on sagittal plane during stance phase, representing the dorsiflexion ability of ankle joint during this phase of gait cycle

minimum value of the ankle joint angle on sagittal plane in stance phase, representing the plantar flexion

peak of ankle dorsiflexion on sagittal plane during swing phase, representing the dorsiflexion ability of ankle joint in this phase of gait cycle

the range of motion at ankle plot on the sagittal plane during the stance phase, calculated as the difference between the maximum and minimum values of the plot in stance phase

the maximum value of ankle moment during terminal stance

the maximum value of generated ankle power during terminal stance (maximum value of positive ankle power during terminal stance) representing the push-off ability of the foot during walking

\section{POST1 session (after six months)}

The effects of the surgical treatment were evident immediately in the first follow-up although rehabilitation 
was not complete. Significant improvements were observed: the speed decreased significantly as well as step length, getting further away from normality, and the duration of stance phase also worsened on the left side.

The knee joint was less flexed with a tendency to hyperextend during stance phase bilaterally and joint excursion increased bilaterally; the knee maximum flexion value in swing (Max Sw index) decreased at a significant level (Fig. 1 b).

The ankle dorsi-plantarflexion plot shifted towards plantarflexion, significantly reducing the high pre-operative dorsiflexion, especially on the left side. The ankle joint was slightly plantarflexed at initial contact and maximum dorsiflexion in stance decreased, on the right side to normal, on the left to mild equinus. In addition, ankle range of motion was reduced. Maximum dorsiflexion in swing decreased, especially on the left side.
Hip flexion during the stance phase decreased and became more physiologic than pre-operatively. Moreover, the range of motion was reduced.

Pelvic anteversion increased, while the pelvic range of motion decreased significantly, getting closer to normal.

Kinetic data revealed a significant reduction of the ankle moment and ankle power generation during terminal stance.

\section{POST2 session (after 12 months)}

Twelve months after surgery, rehabilitation was complete as no more change occurred compared to the following session.

Walking speed increased up to similar pre-operative values. Left stance phase became shorter and step length improved bilaterally.

TABLE II - COMPARISON OF THE SELECTED SPATIO-TEMPORAL PARAMETERS (MEAN AND STANDARD DEVIATION) FOR THE CP SUBJECT IN PRE, POST1, POST2 AND POST3 SESSIONS AND FOR THE CONTROL GROUP (CG)

\begin{tabular}{|c|c|c|c|c|c|c|c|c|c|}
\hline & \multicolumn{2}{|c|}{ Pre } & \multicolumn{2}{|c|}{ Post1 } & \multicolumn{2}{|c|}{ Post2 } & \multicolumn{2}{|c|}{ Post3 } & CG \\
\hline Speed $(\mathrm{m} / \mathrm{s})$ & $0.6(0.1)$ & & $0.3(0.1)^{*}$ & & $0.7(0.1) \downarrow$ & & $0.7(0.1)$ & & $1.2(0.2)$ \\
\hline Step length $(\mathrm{cm})$ & $44.8(5.9)$ & $47.9(2.2)$ & $32.7(1.8)^{*}$ & $28.3(7.8)^{*}$ & $47.7(4.9) \bullet$ & $49.0(2.7) \bullet$ & $48.3(3.5)$ & $47.3(0.8)$ & $54.6(4.2)$ \\
\hline
\end{tabular}

$*=P$-value $<.05$, PRE vs. POST1; $*=P$-value $<.05$ POST1 vs. POST2

TABLE III - COMPARISON OF THE SELECTED KINEMATIC PARAMETERS (MEAN AND STANDARD DEVIATION) FOR THE CP SUBJECT IN PRE, POST1, POST2, AND POST3 SESSIONS AND FOR THE CONTROL GROUP (CG)

\begin{tabular}{|c|c|c|c|c|c|c|c|c|c|}
\hline & \multicolumn{2}{|c|}{ Pre } & \multicolumn{2}{|c|}{ Post1 } & \multicolumn{2}{|c|}{ Post2 } & \multicolumn{2}{|c|}{ Post3 } & CG \\
\hline Mean value & $7.5(2.6)$ & $7.68(3.1)$ & $15.5(0.7)^{*}$ & $14.9(0.5)^{*}$ & $14.1(0.6)$ & $15.2(0.4)$ & $12.4(0.6)$ & $12.2(0.6)$ & $8.8(4.3)$ \\
\hline ROM & $9.5(1.2)$ & $9.7(0.8)$ & $6.5(0.1)^{*}$ & $6.5(0.1)^{*}$ & $9.6(1.7) \diamond$ & $10.5(1.3)$ & $9.9(0.6)$ & $10.9(1.7)$ & $1.6(3.6)$ \\
\hline \multicolumn{10}{|c|}{ Hip Flex-Extension $\left(^{\circ}\right)$} \\
\hline ROM & $34.6(2.9)$ & $34.3(2.3)$ & $24.9(0.4)^{*}$ & $25.4(0.9)^{*}$ & $36.2(0.6)$ & $39.1(2.2)$ & $32.3(1.2)$ & $33.3(1.2)$ & $38.2(5.3)$ \\
\hline \multicolumn{10}{|c|}{ Knee Flex-Extension $\left(^{\circ}\right)$} \\
\hline IC & $49.3(0.4)$ & $44.7(2.0)$ & $-5.8(2.1)^{*}$ & $1.1(2.6)^{*}$ & $9.7(0.3)$ & $0.9(4.0)$ & $5.0(2.2) \bullet$ & $6.3(0.7) \bullet$ & $6.7(5.5)$ \\
\hline $\min S t$ & $53.8(0.7)$ & $44.6(2.2)$ & $-13.6(3.6)^{*}$ & $-6.6(1.1)^{*}$ & $0.5(5.3) \bullet$ & $-14.3(5.9)$ & $-10.0(4.2) \bullet$ & $-3.3(0.3) \bullet$ & $4.2(2.1)$ \\
\hline $\operatorname{Max} \mathrm{Sw}_{\mathrm{w}}$ & $75.4(2.4)$ & $72.2(0.7)$ & $25.5(1.0)^{*}$ & $36.0(1.3)^{*}$ & $32.3(0.3)$ & $34.3(3.5)$ & $39.8(0.9) \bullet$ & $46.6(1.7) \bullet$ & $58.5(4.7)$ \\
\hline $\operatorname{Max} \mathrm{Sw}_{w}$ & $12.5(1.9)$ & $12.4(0.8)$ & $10.9(0.8)$ & $3.1(0.4)^{*}$ & $7.3(0.0)$ & $-1.1(1.1)$ & $6.6(2.6)$ & $2.8(0.6) \bullet$ & $5.8(6.5)$ \\
\hline $\min \mathrm{St}$ & $0.5(0.8)$ & $1.4(1.9)$ & $-2.8(3.6)$ & $-5.8(2.8)^{*}$ & $-2.2(2.2)$ & $-11.6(1.3)$ & $-0.6(3.3)$ & $-6.7(1.5) \bullet$ & $-9.5(5.7)$ \\
\hline ROM St & $24.8(0.7)$ & $26.6(1.9)$ & $15.0(2.1)^{*}$ & $9.0(2.4)^{*}$ & $17.4(1.2)$ & $20.0(0.6)$ & $10.3(3.1) \bullet$ & $17.6(0.9) \bullet$ & $23.4(4.8)$ \\
\hline
\end{tabular}

${ }^{*}=P$-value $<.05$, PRE vs. POST1; $\bullet=P$-value $<.05$ POST1 vs. POST2; $\bullet=P$-value $<.05$ POST2 vs. POST3. Abbreviations: IC: initial Contact; Max: Maximum value; min: minimum value; St: Stance phase; Sw. Swing phase; ROM: Range of Motion. 
Knee flexion increased on the right side, while the left side the recurvatum increased in midstance (Fig. 1 c).

The ankle joint showed a more physiologic pattern bilaterally, even if the left side remained in a plantarflexed position at initial contact; its range of motion improved to a significant level.

Mean pelvic position did not modify significantly but showed a slight increase in its range of motion. The hip joint improved significantly. The pattern was physiologic.

Maximum ankle moment and power increased at terminal stance improving propulsion.

\section{POST3 session (after 18 months)}

\section{Clinical assessment}

The patient walked upright. The knee extended fully passively as well as actively. Hip extension improved to a normal extension of $10^{\circ}$ spontaneously.

\section{Gait analysis}

No significant changes occurred for the spatio-temporal parameters and at the proximal joints (such as pelvis and hip joints).

Regarding the knee position a more physiologic and symmetric gait was attained at initial contact; the recurvatum position was maintained in midstance and it was more accentuated in comparison to the previous session. The maximum flexion in swing was higher than POST2 assessment, causing an improvement in terms of knee joint excursion on both sides (Fig. 1 d).

The ankle joint position improved especially on the left side (IC and Max Sw indices); a bilateral reduction in terminal stance plantarflexion was present with a reduction in their range of motion.

Kinetic ankle parameters did not modify their values.

\section{DISCUSSION}

Severe crouch gait in patients with spastic diplegia causes excessive loading of the patellofemoral joint and may result in anterior knee pain, gait deterioration, and gradual loss of function. In the last few years the use of patellar tendon shortening procedure appears to give good results for the treatment of crouch gait, but to the best of our knowledge the literature on this procedure has little evidence.

From the clinical need to improve the knowledge on the effects of this treatment, in this case study we quantitatively assessed the effects of patellar tendon shortening for the management of crouch gait using GA. This case of a teenager with diplegia secondary to $\mathrm{CP}$ presents with persisting crouch in spite of adequate soft tissue lengthening, which resulted in an almost complete passive knee extension. The patient, however, did not show any benefit concerning gait. As a reason for the remaining problem, overlength of the knee extensors and the Achilles' tendons were identified with the latter less pronounced. Consequently, the knee extensors were shortened. The quantitative effect of these interventions on the gait pattern was documented and quantified throughout four sessions (PRE, POST1, POST2, and POST3 sessions).

Analysis of the PRE session data pointed out anomalous spatio-temporal parameters, non-physiologic kinematics of hip and knee joints through the entire gait cycle, connected to hamstrings spasticity, and quite normal ankle pattern shifted towards dorsiflexed position, because of a weak and long triceps. A satisfactory magnitude of maximum ankle moment and reduced push-off ability in terminal stance was evident bilaterally. Sagittal hip kinematics revealed a constant flexion which corresponded to the hip flexion contracture in the clinical assessment.

Six months after surgery (POST1 session) the treatment caused a significant reduction of the excessive hip and knee flexion bilaterally; it is important to notice the tendency to recurvatum in this session on both sides, and that the hip flexion deformity resolved spontaneously. The ankle dorsi-plantarflexion plot shifted towards plantarflexion showing a less normal shape, with a reduction in range of motion bilaterally. A significant reduction in propulsion ability during terminal stance was also evident.

In POST2 session a more normal knee position at initial contact and a normalization of ankle pattern occurred, especially on the right side. Ankle kinetics revealed a bilateral improvement in ankle moment and ankle power, demonstrating better push-off ability during walking.

Eighteen months after surgery the patient showed

TABLE IV - COMPARISON OF THE SELECTED KINETIC PARAMETERS (MEAN AND STANDARD DEVIATION) FOR THE CP SUBJECT IN PRE, POST1, POST2 AND POST3 SESSIONS AND FOR THE CONTROL GROUP (CG)

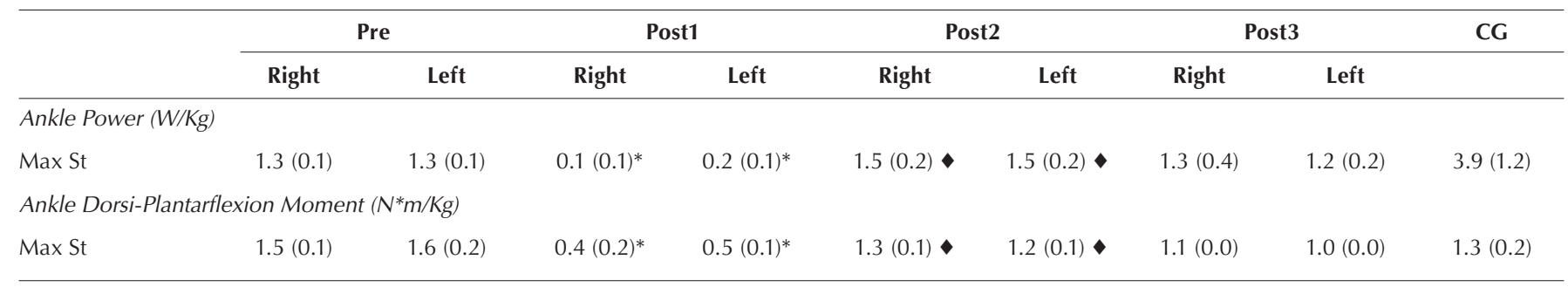

$*=P$-value $<.05$, PRE vs. POST1; $* P$-value $<.05$ POST1 vs. POST2. Abbreviations: Max: Maximum value; St: Stance phase. 
a more physiologic gait and lower limb joint kinematics improved overall; only a mild knee hyperextension remained. The improvements in ankle push-off lasted over time. The hip flexion contracture disappeared spontaneously.

\section{CONCLUSIONS}

The results presented in this case report demonstrated that especially in severe cases, both sides of the joint need to be addressed. Knee extensor shortening presents a reasonable option to treat crouch gait in patients with $\mathrm{CP}$, especially in case of failure of prior surgery. Full passive knee extensibility, however, is a prerequisite for adequate shortening. Significant kinematics improvements were evident over the follow-up period. Maximal knee and ankle moments remained at their pre-operative value. However, it is important to highlight that the main aim of surgery was to improve knee kinematics in order to increase the patient's range of function and independence in the community and not to increase ankle push-off capacity by reducing crouch gait. The importance of hip flexion contractures should not be overestimated.

Our results could not be compared with previous research as there is little literature on this topic. To the best of our knowledge only one study quantitatively assessed the effects of this technique (5). However, while in that paper the authors transposed the tibial tubercule distally, in this case study the patellar ligament was duplicated and thus shortened which seems to be more convenient in the growing skeleton; for this reason the comparison loses meaning.

Future studies should be performed to confirm these results with a larger sample that might give the opportunity for more in depth investigation together with stronger statistical findings; however, these data demonstrated that the technique proposed (patellar tendon shortening) may represent an encouraging treatment for severe crouch gait in $\mathrm{CP}$.

\section{Financial support: None.}

\section{Conflict of interest: None.}

Address for correspondence:

Veronica Cimolin

Department of Electronics, Information and Bioengineering

Politecnico di Milano

Via Golgi 39

20133 Milano, Italy

veronica.cimolin@polimi.it

\section{REFERENCES}

1. Gage JR. The treatment of gait problems in cerebral palsy. Gage JR editor. London: Cambridge University Press; 2004. Treatment principles for crouch gait; 382-97.

2. Hicks J, Arnold A, Anderson F, et al. The effect of excessive tibial torsion on the capacity of muscles to extend the hip and knee during single.limb stance. Gait Posture 2007; 26: 546-52.

3. Stout JL, Gage JR, Schwartz MH, et al. Distal femoral extension osteotomy and patellar tendon advancement to treat persistent crouch gait in cerebral palsy. J Bone Joint Surg Am 2008; 90: 2470-84.

4. Wangjam K, Singh AJ, Singh LN. Management of crouch in cerebral palsy diplegia. IJPMR. 2005; 16: 12-15.

5. Rodda JM, Graham HK, Nattrass GR, et al. Correction of severe crouch gait in patients with spastic diplegia with use of multilevel orthopaedic surgery. J Bone Joint Surg 2006; 88: 2653-64.

6. Young JL, Rodda J, Selber P, et al. Management of the knee in spastic diplegia: what is the dose? Orthop Clin North Am 2010; 41: 561-77.

7. Davis R B, Ounpuu S, Tyburski D J, et al. A gait analysis data collection and reduction technique. Hum Mov Sci 1991; 10: 575-87. 